\title{
Accuracy of non-invasive and minimally invasive hemodynamic monitoring: where do we stand?
}

\author{
Issa Pour-Ghaz ${ }^{1}$, Theodore Manolukas ${ }^{1}$, Nathalie Foray ${ }^{2}$, Joel Raja ${ }^{1}$, Aranyak Rawal ${ }^{1}$, Uzoma N. Ibebuogu ${ }^{3}$, \\ Rami N. Khouzam ${ }^{3}$ \\ ${ }^{1}$ Department of Internal Medicine, ${ }^{2}$ Department of Medicine - Critical Care, ${ }^{3}$ Department of Internal Medicine, Division of Cardiovascular \\ Diseases, University of Tennessee Health Science Center, Memphis, TN, USA \\ Contributions: (I) Conception and design: I Pour-Ghaz, RN Khouzam; (II) Administrative support: UN Ibebuogu, RN Khouzam; (III) Provision \\ of study materials or patients: None; (IV) Collection and assembly of data: I Pour-Ghaz; (V) Data analysis and interpretation: I Pour-Ghaz; (VI) \\ Manuscript writing: All authors; (VII) Final approval of manuscript: All authors. \\ Correspondence to: Issa Pour-Ghaz, MD. Internal Medicine Resident Physician, PGY-2, University of Tennessee Health Science Center, 956 Court \\ Ave., Suite H314, Memphis, TN 38163, USA. Email: ipourghaz@gmail.com.
}

\begin{abstract}
One of the most important variables in assessing hemodynamic status in the intensive care unit (ICU) is the cardiac function and blood pressure. Invasive methods such as pulmonary artery catheter and arterial line allow monitoring of blood pressure and cardiac function accurately and reliably. However, their use is not without drawbacks, especially when the invasive nature of these procedures and complications associated with them are considered. There are several newer methods of noninvasive and minimally invasive hemodynamic monitoring available. In this manuscript, we will review these different methods of minimally invasive and non-invasive hemodynamic monitoring and will discuss their advantages, drawbacks and limitations.
\end{abstract}

Keywords: Hemodynamic monitoring; minimally invasive; non-invasive; continuous monitoring; cardiac output

Submitted Jun 16, 2019. Accepted for publication Jun 28, 2019.

doi: 10.21037/atm.2019.07.06

View this article at: http://dx.doi.org/10.21037/atm.2019.07.06

\section{Introduction}

One of the most important variables for patient assessment in the intensive care unit (ICU), hospital, or clinic is the ability to measure vital signs accurately. Among these vital signs, blood pressure and cardiac function serve as important markers of cardiovascular system, thus having reliable methods of measurement play a vital role. Blood pressure correlates directly with the cardiac and vascular function and it can be used to assess the cardiac output. Blood pressure fluctuations can lead to devastating outcomes if not controlled well. Hypotension has been linked to postoperative ischemic stroke, myocardial injury, and acute kidney injury $(1,2)$. In a recent cohort study of intraoperative blood pressures, when the mean arterial blood pressure was less than $55 \mathrm{mmHg}$, even if for a short time, it was associated with development of acute kidney injury and myocardial injury (3). It has been demonstrated that mortality risk was increased by 1.036 times per minute of systolic blood pressure less than $80 \mathrm{mmHg}$ (4). In a recent study by Mascha et al., researchers were able to show that variability in mean arterial pressure independent of time-weighted average of the mean arterial pressure, was associated with 30 -day post-operative mortality in patients who underwent noncardiac surgery (5). Intraoperative systolic blood pressure variability in patients who underwent aortocoronary bypass surgery has been shown to result in increased 30-day postoperative mortality, which directly correlated with the amount of time per minute outside of the target blood pressure range (6). As a result, management of blood pressure is beneficial for increasing long term benefits and decreasing morality.

There are several methods that can be utilized for measurement of blood pressure and cardiac output 
non-invasively, minimally invasively and noninvasively. Oscillometric non-invasive method is the oldest method of hemodynamic monitoring that uses an air-filled pressure cuff. However, due to a wide degree of unreliability and inaccuracy, these devices are not reliable in acute conditions and do not provide reliable information about the cardiac function (7-9). Pulmonary artery catherization (PAC) is an invasive method for close hemodynamic monitoring during surgery and ICU stay that has been used for more than four decades. It allows for collection of data regarding cardiac pressures, volume status and oxygen saturation (10). Use of PAC involves a relatively invasive insertion of a large-bore multi-lumen catheter to the pulmonary artery which can lead to complications such as pulmonary artery rupture, right bundle-branch block, complete heart block, and catheter related sepsis (11). Despite its well-known limitations and complications associated with it, it remains the gold standard (12).

Arterial cannulation is a minimally invasive method of hemodynamic monitoring which when coupled with the newer devices, such as FloTrac (Edwards Lifesciences, Irvine, California) system, can provide information regarding blood pressure and cardiac function. This type of cannulation is usually done on the radial or brachial arteries, although other sites can be used. This method is usually chosen when other non-invasive methods cannot be used, for example in the cases of severe hypotension, severe trauma leading to multiple fractures, or patients who have multiple comorbidities (13). One advantage of arterial cannulation is that it is possible to use this access to obtain frequent blood gas specimen, which are useful in the ICU setting where frequent laboratory tests and arterial sampling are needed. Some contraindications to using an arterial cannulation include poor peripheral perfusion and absent pulses (14). Some of the other complications include vessel occlusion, hematoma, infection, and ischemic damage (15).

The other category of hemodynamic monitoring is the noninvasive continuous methods of monitoring by using finger cuff devices such as the CNAP (CNSystems Medizintechnik, Graz, Austria) system which can calculate cardiac output and blood pressure by pulsation and pressure waveform analysis in the digital arteries. These devices have shown promising results in cardiac monitoring in surgical cases and non-critical cases while showing good promise when used as a transitioning tool in critically ill until more advanced hemodynamic monitoring can be obtained (16).

In this manuscript we will briefly discuss the non-invasive intermittent blood pressure measurement techniques and will focus heavily on minimally invasive and non-invasive continuous hemodynamic monitoring.

\section{Non-invasive intermittent hemodynamic monitoring}

This is by far the most common method of measuring blood pressure both in outpatient and inpatient settings. It involves an air-filled cuff that can measure the blood pressure manually (by the operator) and automatically (by the device). When measured by the operator, it can be done by palpation or by auscultation. In the palpation method, the operator palpates the, for example, the radial artery and deflates the cuff. The pressure where the pulses are felt is the systolic pressure. The advantage of this method is the benefit of it being quick and the absence of the need for a stethoscope, but it does only provide the systolic blood pressure. When the auscultation method is used, the pressure of the cuff is increased beyond the systolic blood pressure. Stethoscope is placed on the artery and Korotkoff sounds are auscultated. The first sounds correspond to the systolic blood pressure while the last sounds correspond with the diastolic blood pressure. This technique requires the operator to be trained well. The automated system using a pressure cuff applies the same technique, inflating the cuff to a preset pressure and decreasing the pressure slowly. The oscillations are detected by the machine corresponding to systolic and diastolic blood pressures. Mean arterial pressure corresponds to the maximum oscillations. The algorithms used by these machines are proprietary and dependent on each manufacturer. These machines base the systolic and diastolic pressure on these oscillations (17).

When comparing the oscillometric noninvasive and invasive arterial blood pressure measurements, it has been established that noninvasive techniques underestimate blood pressure during hypertension and overestimates during hypotension (18). Due to the morbidity and mortality in severely ill patients and the demonstrated drawbacks of inadequate measurement of blood pressure, such as acute kidney injury and ICU mortality, more well developed and reliable techniques would be beneficial for monitoring critically ill patients (19). Non-invasive intermittent techniques are therefore more suitable when patient is out of the window for hemodynamic instability or lack of the need for close monitoring (i.e., not undergoing surgery or not in the ICU). Even though traditional intermittent blood pressure measurement is reliable for outpatient and noncritical inpatient settings, the readings are highly dependent 
on the use of proper cuff size. Therefore, making sure the actual measurement is proper for patient is of high value (20). It has been demonstrated that improper size and location of cuff used for measurement of blood pressure could lead to inadequate management of blood pressure in patients $(8,21,22)$.

\section{Minimally invasive and non-invasive hemodynamic monitoring}

Close hemodynamic monitoring in critically ill patients are of paramount value and as the available devices become more advanced, ability to perform this monitoring moves towards a more non-invasive method. An optimal tool would be able to give 'gold standard' level of monitoring while staying non-invasive. In most ICU settings, use of pulmonary artery catheter (PAC) together with an arterial line which utilize waveform analysis are currently the gold standards in practice. However, complications such as bleeding, infection and dependence on good peripheral vascular in addition to being cost intensive and limited availability limits their use (23).

\section{Minimally invasive hemodynamic monitoring}

For critically ill patients, continuous hemodynamic monitoring is a matter of life and death. Basic vital signs such as blood pressure, heart rate and peripheral oxygen saturation can be easily measured in a non-invasive manner. For a patient in the ICU, another set of variables, such as cardiac output (CO) and systemic vascular resistance (SVR) are crucial. Continuous hemodynamic monitoring is used to guide many intensive care unit therapies, particularly the use of vasopressors. The gold standard for the measurement of CO is the pulmonary artery catheter (PAC, Swan-Ganz catheter) (24). The calibrated PAC is placed through the jugular or subclavian vein and is threaded through the right atrium to the right ventricle and lodged in a distal branch of the pulmonary artery. From this location a plethora of measurements can be obtained. Right atrial pressure, pulmonary capillary wedge pressure and $\mathrm{CO}$ can all be obtained from a PAC. The PAC is no longer the most used technique for continuous monitoring due to its highly invasive nature. More commonly transpulmonary thermodilution is used to measure continuous CO. This technique requires a central venous catheter and an arterial line. In transpulmonary thermodilution, a cold fluid bolus is injected through the central line, the Stewart Hamilton equation is then used to calculate the CO. By using an algorithm based on the analysis of the arterial pulse contour continuous $\mathrm{CO}$ can be obtained (24). Both above techniques require central access which comes with several risks to the patient, most notably infection.

In contrast to PAC, newer systems rely on limited invasive arterial lines to give comparable information regarding the hemodynamics. There are multiple minimally invasive methods available to monitor cardiac output and stroke volume. Here we will discuss three such systems which use minimally invasive access for estimation of cardiac output, cardiac index and providing information about the hemodynamics.

\section{PiCCO}

PiCCO (Pulsion Medical Systems, Munich, Germany) system is one which uses 2-element Windkessel model to calculate the cardiac output, stroke volume and arterial pressure waveform. It works on the principle that the amount of flow into the system must equal the amount of outflow and that compliance of the vessel affects the flow. Hence, during systole increased pressure in the vessel causes expansion and distention which is expelled during diastole as pressure decreases. The cardiac output and aortic compliance are measured by using transpulmonary thermodilution and arterial waveform is used for obtaining pressures (25). Souto Moura and colleagues in a recent study demonstrated that the PiCCO system when compared to echocardiography for assessment of cardiac output estimation and concordance, was able to produce values in high agreement in normothermic patients (26). One limitation of the PiCCO system is its needs of transpulmonary measurement and its invasive nature. Recent studies have shown that when more distant central lines are used, the device is not able to predict the cardiac function reliably (27). Despite its limitations, $\mathrm{PiCCO}$ is extremely effective in goal directed therapy of patients in ICU and early fluid resuscitation efforts, thereby, decreasing mortality (28). Compared to PAC, PiCCO method combines pulse contour analysis and transpulmonary thermodilution techniques and has been shown to be superior to PAC in hemodynamic monitoring of critically ill patients $(29,30)$.

\section{FloTrac/Vigileo}

FloTrac (Edwards Lifesciences, Irvine, California) system is a system that enables continuous cardiac output (CO) measurement by using pulse rate and stroke volume. To 
calculate the stroke volume (SV), arterial pulse pressure (PP) standard deviation is calculated by sampling arterial pressure data points at $100 \mathrm{~Hz}$ for 20 seconds, which is used to calculate the standard deviation around these points. Then a conversion factor is applied which represents calculated systemic vascular resistance, arterial compliance and modifiers such as age, sex, body weight and height are taken into account (25). FloTrac system has the ability to continuously update and reproduce the hemodynamic parameters such as $\mathrm{CO}$, cardiac index (CI), stroke volume variation (SVV), SV, stroke volume index (SVI), and in contrast to PiCCO method, does not require recalibration (31). SVV has been shown to be a reliable indicator for fluid optimization especially in post-op patients as a parameter for adequate volume resuscitation for prevention of acute kidney injury (32). Button and colleagues compared this system with PiCCO and PAC to evaluate cardiac output measurements in patients undergoing cardiac surgery and they showed that the results obtained by all three systems were comparable, making FloTrac a suitable alternative for $\mathrm{CO}$ measurement especially in light of its less invasive nature (33). FloTrac system is significantly less invasive and has consistently shown lower rates of complications (12). This ability of FloTrac to trend $\mathrm{CO}$ is continuously improving with newer generations of the device (34). One major limitation of this device is its ability to accurately estimate $\mathrm{CO}$ in patients who are morbidly obese (35). Another group of patients who have been shown to have unreliable readings in CI when compared to PAC method are the patients undergoing liver transplantation (36). Maeda and colleagues point out a major limitation of the FloTrac/Vigileo system is being its inability to reliably measure CI in patients with high or low SVR and in patients with low CO. They demonstrated that in patients undergoing abdominal aortic aneurysm repair the degree of accuracy was not acceptable. They argue that the surge in SVR during aortic clamping, hemodynamic instability and low cardiac output contribute to this phenomenon (37). The deleterious effects of low CI and high SVR on measurements obtained by this system has been reported by other authors $(38,39)$. There have been some updates with the newer generation of the device that enable the system to respond faster to the changing vascular tone in patients, however, these upgrades have not eliminated all the limitations $(40,41)$. Main advantage of the FloTrac system is its ability to be able to be connected to a general peripheral arterial catheter and display the arterial information while a Vigileo monitor addition will give us the CO, SV and SVV. This system works both with peripheral and central arteries and in emergency situations femoral arteries can give more accurate information (42). The correlation between radial and femoral arteries was tested during bypass periods which showed good correlation (43). Especially since FloTrac refreshes its calculations every 20 seconds, it performs measurements nearly in real time. The accuracy of this system has been demonstrated in patients with normal CI and can be used as a reliable method for monitoring in such patients (44). It has also been used for monitoring patients undergoing intraoperative chemotherapy with good results (45).

\section{LiDCO}

LiDCO (LiDCO, London, United Kingdom) system uses pulse power analysis to determine $\mathrm{CO}$ and lithium is used as an indicator. It runs two algorithms: LiDCO system which is an indicator dilution monitoring system and PulseCO system which is a continuous arterial waveform analyzing system (46). Lithium transit time and concentration curve based on injection time from venous to arterial system is used to determine the CO (25). Thus, it is recommended that the PulseCO to be recalibrated every 8 hours or when there is a major hemodynamic change. Hence, this system only needs an arterial line and peripheral line. LiDCO system can provide parameters such as $\mathrm{CO}$, mean arterial pressure (MAP), SVR, SV, SVV and pulse pressure variation (PPV). Donati and colleagues compared the LiDCO to PiCCO system and reported acceptable correlation between the measurements of CI (47). Similar findings have also been reported by other authors, where the LiDCO system does produce value with close correlation to the other minimally invasive systems, but it did tend to underestimate the CI when CIs were especially high $(48,49)$. The main drawback they reported was the need for repeated recalibration of the system and it being more likely to be affected by arterial spasm and changes in compliance. Cross validity of the $\mathrm{CO}$ and SVV measurements between these devices have also been questioned recently due to the vastly different methods used by each system to calculate the CO. Flotrac/Vigileo system uses arterial pressure waveform and applies various factors when calculating CO and SVV, while $\mathrm{LiDCO}$ is based on PulseCO algorithm and lithium dilution, hence lack of agreement between readings may represent the basic fundamental difference in principles for measurement (50).

Overall, these devices have enabled close monitoring of patients and detection of hemodynamic instability in at risk 
patients and timely management of different conditions, but have not been able to replace PAC method in all cases, especially in cardiac surgery cases $(51,52)$. Geisen and colleagues demonstrated the limitation of LiDCO, PiCCO and FloTrac systems in providing reliable readings during the postoperative period when compared to intermittent transpulmonary thermodilution technique (53). Even though each of these systems come with their own set of limitations, the wide selection does allow application of specific monitoring devices to individual settings based on clinical judgment.

\section{Non-invasive bemodynamic monitoring}

One of the areas of recent advancement in the field of blood pressure monitoring has been the continuous improvement in noninvasive continuous blood pressure measurement. These devices allow for real-time measurement of blood pressure and assessment of the patient. There are two main techniques used for measuring blood pressure which are applanation tonometry and volume clamp (VC) method.

\section{Applanation tonometry}

Applanation tonometry (AT) allows continuous measurement of $\mathrm{CO}$ by using a transducer which is strapped to an artery with a bone underneath. The artery is then flattened making the transmural pressure zero which allows for the arterial pulse wave to be obtained (54). This allows for detailed measurement of the systolic and diastolic arterial pressures and assessment of the central vascular pressures (55). The effectiveness of the applanation tonometry has been shown in measurement of the brachial artery blood pressure for management of hypertension, measuring radial artery pressure for optimal management of obstructive sleep apnea, measurement of pulse pressure as a predictor of left ventricular mass progression, earlier identification of individuals at risk for diastolic dysfunction, and identification of coronary artery disease with its severity (56). One of the systems used is called T-Line (Tensys Medical, San Diego, CA, USA) system which determines a waveform signal and $\mathrm{CO}$ is measured continuously by analyzing the pulse wave and taking into account data points such as sex, age, height, body weight and blood pressure variables (57). Studies have demonstrated that the T-Line system can measure blood pressure accurately with good readings when compared to the arterial catheter (58-60). Sun and colleagues assessed the reliability and precision of the applanation tonometry device TL-300 in 30 patients undergoing colon surgery. They found strong linear correlation between the SBPs and MBPs and good correlation in DBPs providing them reliable readings when compared to the arterial puncture method, the result of which argued for dependable use of this device in arterial pressure measurement when invasive measurement of arterial pressure is not needed or contraindicated (61). Lin et al. showed that in patients undergoing elective neurosurgery, TL-300 system was able to be used for accurate and precise blood pressure monitoring intraoperatively when compared to invasive methods (62). DMP-Life system is another system that uses AT method by using 5 piezoresistive semiconductor transducer sensors after maximal pulse pressure is identified to calculate the SV based on the systolic area of blood pressure waveform and demographic data and has shown to have reasonable accuracy and precision $(63,64)$.

However, there are studies which demonstrate that the precision and accuracy of the device is not always guaranteed. This was especially true in critically ill patients in ICU settings whereby different measurements were obtained when the arterial line was compared to the applanation tonometry readings particularly in those with poor arterial pulse pressures (65). A major limitation of the system has been the inability to reliably calibrate the device by non-invasive methods, whereby the measurement accuracy was affected when invasive and non-invasive methods of calibration were used (66). Another limitation is that measurements are easily affected by use of vasoactive supplements and motion $(56,58)$. Nevertheless, the newer generation of the applanation tonometry have increasingly better measurements which agree with the readings in arterial line method. With more widespread use of the device and more awareness, its use can lead to better outcomes in management of chronic conditions such as heart failure or high blood pressure.

\section{VC method}

VC measures blood pressure noninvasively by utilizing an inflatable cuff which adjusts automatically to measure the arterial blood pressure waveform over the full pulse based on the Peňáz principle. In this cuff, there is an infrared photodiode and light detector that measures the diameter of the finger artery (54). It is the absorption of the infrared through the finger that is used to measure the arterial volume, hence the pressure. This cuff pressure adjusts rapidly to clamp the arterial volume throughout the pulse to be able to measure the waveform pressure and pulse 
contour (18). Cuff pressure is constantly adjusted during the cardiac cycle to keep the volume in the finger constant and these adjustments over time are used to create the blood pressure waveform and analyze the pulse wave (67). Thus, different pathophysiological changes affect the arterial compliance and resistance, therefore the waveform. Two of the systems currently available are the Clear-Sight system (Edwards Lifesciences, Irvine, CA) and the CNAP system (CNSystems Medizintechnik, Graz, Austria).

\section{ClearSight}

ClearSight (Edwards, Irvine, California, USA), working based on the modified Peňáz principle using Nexfin technology, applies an enhanced vascular unloading technology to measure arterial blood pressure continuously and noninvasively through application of finger cuff technology which is able to provide information regarding heart rate, $\mathrm{SV}, \mathrm{CO}$, CI, SVI, SVV, SVR and SVR index (68). The disposable pneumatic finger cuff continuously inflates and deflates throughout the cardiac cycle according to the signal it receives via the photoplethysmography sensor which detects changes in light absorption in the artery during pulsation which increases during systole and decreases in diastole. The absorption detected has direct correlation with the arterial blood volume. By dynamically changing the pressure in the cuff, the system can counteract the pressure changes in the artery, thus keeping a constant finger volume, i.e. $\mathrm{VC}$ technique. By applying an algorithm, VC can quantify the cardiac parameters. In this system, no cuff calibration is needed, and the system automatically recalibrates every 5-70 beats to compensate for ongoing changes in vascular system $(69,70)$.

ClearSight was used in a randomized trial for continuous monitoring of blood pressure to see its effectiveness in detection of hypotensive episodes during surgery. It was demonstrated that the use of continuous blood pressure monitoring lead to earlier detection of hypotensive episodes during surgery and more timely treatment (71). There have been multiple studies that have compared the $\mathrm{CO}$ from the ClearSight device to PiCCO and PAC, the current gold standard in continuous hemodynamic monitoring (23). Comparison between Clearsight and either PAC or PiCCO was shown as level concordance which was calculated as the percentage of the pairs of reading with the same direction of change. The concordance between Clearsight and the other measurement should be greater than $90 \%$. ClearSight was shown to be accurate, with an $84-100 \%$ level of concordance, meaning that the ClearSight CO readings would change in the same direction as the gold standard measurement. ClearSight is also able to show the magnitude of change of CO, with $84-89 \%$ of data points within $+/-1.0 \mathrm{~L} / \mathrm{min}$ limits of agreement terms (23). Sakai and colleagues investigated the reliability of ClearSight system compared to FloTrac in patients undergoing laparoscopic radical prostatectomy and reported that the measurement did have close correlation and could be used interchangeably, even though large difference between the values obtained was noted (72). The reliability and feasibility of continuous monitoring in cardiac surgery patients and good within subject precision have also been demonstrated $(73,74)$. Ameloot and colleagues performed an observational study in 45 patients reported good CO correlation when they compared to transpulmonary thermodilution and continuous femoral arterial pulse contour derived $\mathrm{CO}$ by the PiCCO. They reported that it was most accurate in patients with high CO and low SVRI and least accurate in patients who had a low CO and high SVRI. They also note that the device was easy to use and apply to patients and not a substantial amount of training was needed. They viewed the device as one that could be applied in the ICU and one that would serve as a great bridge to more advanced invasive monitoring system in critically ill patients (75). CO measurements have also been validated against echocardiography which has shown reasonable agreement (76). Its safety in reliable perioperative monitoring has also been demonstrated by other authors (77). Aritürk and colleagues have demonstrated that it can be used for monitoring for uncomplicated hemodynamically stable patients in ICU who have undergone coronary artery bypass graft when it was compared to FloTac/Vigileo and echocardiography (78). One big advantage of the ClearSight system was the very low risk of complications such as infection or thrombus which are associated with the invasive systems (69). These findings have made it a suitable monitor for perioperative continuous measurements of CO.

While ClearSight can admirably measure $\mathrm{CO}$, it is unlikely that it can completely replace thermodilution, which is the gold standard. There have been several studies comparing CO obtained by different measurement techniques, most notably Critchley and Critchley method, which relies on a percentage of error of $30 \%$ or less when comparing two readings for $\mathrm{CO}$ from two different devices. When looking specifically at the pulse contour method of calculating CO, which is used by ClearSight, a metaanalysis of 16 studies showed a weighted percentage of error of $46.4 \%$ (79). Based on the available data, CO monitoring 
by ClearSight is not equivalent to the gold standard and the two measurements cannot be used interchangeably (80). This does not mean that ClearSight is not a clinically valuable tool, it can be used effectively to monitor CO and blood pressure is critically ill patients who are not appropriate for invasive monitoring.

\section{CNAP}

CNAP device (CNSystems Medizintechnik AG, Graz, Austria) uses vascular unloading technique based on the Peňáz principle whereby infrared light is transmitted through the finger where the artery is located and based on the absorption, CNAP calculates the arterial blood pressure (54). Finger cuff constantly adjusts the pressure and blood pressure waveforms obtained from the CNAP system is calibrated by taking into account values obtained from an upper arm cuff, then proprietary algorithms are applied to calculate areas under the cure for the systolic and diastolic pressures $(81,82)$. CNAP provides beat-tobeat blood pressure readings which have been used for a multitude of procedures including vascular surgery, rapid ventricular pacing during transaortic valvular replacement procedure, and bariatric surgery (83-85). Its accuracy was tested in ICU by Smolle et al. which showed good capability to trend the blood pressure in critically ill patients (86). Wagner and colleagues studied the applicability of CNAP in acutely ill emergency department patients and reported that it allows for immediate recognition of clinically relevant hypotensive episodes while showing a reasonable agreement with intermittent oscillometric measurements. They emphasize that its use is particularly suitable for patients who do not have any clear indication for use of invasive techniques, thereby serving as a suitable bridge (87). Biais and colleagues compared blood pressure findings in 35 stable surgical patients by using CNAP and arterial line method to assess their fluid responsiveness which performed satisfactorily (88). Dewhirst and colleagues assessed the function of CNAP when the patients were placed in prone position and compared the readings to arterial line measurements and determined that mean arterial pressure readings were in agreement with guidelines and the DBP readings closely correlated with the standards, SBP measurements were least accurate (89). There are specific cases where placement of arterial line cannot be achieved, thus CNAP can serve as a valid alternative when time is limited (16). Jagadeesh et al. demonstrated that percentage within limits of agreement was $94.5 \%, 95.1 \%$ and $99.4 \%$ for SBP, DBP, and MAP making CNAP a reliable alternative for non-invasive continuous monitoring device with results that are comparable with invasive counterparts (90). However, the main limitation for these devices is the need for good peripheral pulse pressure to measure the blood pressure and in hemodynamically unstable patients such reliable readings cannot be guaranteed (86).

One of the most important advantages of such devices is the ability to use these devices without having to worry about major complications. They can provide vital information without putting the patient at risk. Meanwhile, like any other method, they do have some limitation. These methods are sensitive to movements by the subject. Their use is also limited in patients who have severe vasoconstriction or severe peripheral vascular disease. Hence, their use will be limited according to the patient population (17). There have been multiple studies comparing the invasive and non-invasive methods of blood pressure measurement and have demonstrated their noninferiority $(91,92)$. They can reliably monitor blood pressure and can measure cardiac output which is vital in monitoring critically ill patients $(23,93)$. Thus, they do offer a valid alternative to invasive methods in non-critical patients or as a transition to invasive methods until they can be secured.

\section{Conclusions}

When it comes to blood pressure monitoring, arterial cannulation and PAC are the gold standards in acute and critical care settings. These are invasive procedures that provide accurate hemodynamic monitoring when compared to the non-invasive means. However, they are not always necessary and sometimes relatively contraindicated in some patients, for example in those who are anticoagulated or have a coagulopathy. Non-invasive methods are a great and safe alternative for hemodynamic monitoring in patients who are in critical or non-critical state. These devices have less complications, are less expensive and can be used in patients who need more in-depth monitoring of their hemodynamic status. Thus, their use with time will be more widespread as the awareness increases. Their reliability has constantly increased with time and advancements in the newer generation of devices. These devices can serve as a great alternative to invasive monitoring of blood pressure in patients who are out of the critical stage or undergoing elective procedures. While intermittent blood pressure monitoring might be suitable in stable patients, use of the non-invasive continuous methods can be more appropriate in close monitoring of patients who are at risk of hemodynamic compromise or where the invasive methods 
would put patients at unnecessary increased risk. In critically ill patients, use of minimally invasive and invasive methods for monitoring are still recommended.

\section{Acknowledgments}

None.

\section{Footnote}

Conflicts of Interest: The authors have no conflicts of interest to declare.

Ethical Statement: The authors are accountable for all aspects of the work in ensuring that questions related to the accuracy or integrity of any part of the work are appropriately investigated and resolved.

\section{References}

1. Bijker JB, Persoon S, Peelen LM, et al. Intraoperative hypotension and perioperative ischemic stroke after general surgery a nested case-control study. Anesthesiology 2012;116:658-64.

2. Salmasi V, Maheshwari K, Yang D, et al. Relationship between intraoperative hypotension, defined by either reduction from baseline or absolute thresholds, and acute kidney and myocardial injury after noncardiac surgery a retrospective cohort analysis. Anesthesiology 2017;126:47-65.

3. Walsh M, Devereaux PJ, Garg AX, et al. Relationship between intraoperative mean arterial pressure and clinical outcomes after noncardiac surgery toward an empirical definition of hypotension. Anesthesiology 2013;119:507-15.

4. Monk TG, Saini V, Weldon BC, et al. Anesthetic management and one-year mortality after noncardiac surgery. Anesth Analg 2005;100:4-10.

5. Mascha EJ, Yang D, Weiss S, et al. Intraoperative mean arterial pressure variability and 30-day mortality in patients having noncardiac surgery. Anesthesiology 2015;123:79-91.

6. Aronson S, Stafford-Smith M, Phillips-Bute B, et al. Intraoperative systolic blood pressure variability predicts 30-day mortality in aortocoronary bypass surgery patients. Anesthesiology 2010;113:305-12.

7. Lakhal K, Ehrmann S, Boulain T. Noninvasive BP monitoring in the critically ill time to abandon the arterial catheter? Chest 2018;153:1023-39.

8. Watson S, Aguas M, Bienapfl T, et al. Postanesthesia patients with large upper arm circumference: is use of an "extra-long" adult cuff or forearm cuff placement accurate? J Perianesth Nurs 2011;26:135-42.

9. Mirdamadi A, Etebari M. Comparison of manual versus automated blood pressure measurement in intensive care unit, coronary care unit, and emergency room. ARYA Atheroscler 2017;13:29-34.

10. Gupta A, Mishra S. Non-invasive cardiac output monitoring - To be or not to be, that is the question! Indian Heart J 2017;69:293.

11. Evans DC, Doraiswamy VS, Prosciak MP, et al. Complications associated with pulmonary artery catheters: A comprehensive clinical review. Scand J Surg 2009;98:199-208.

12. Botsch A, Firstenberg MS. Comment on the Edwards FloTrac/Vigileo versus pulmonary artery catheter study: What is really going on with this patient? Int J Crit Illn Inj Sci 2017;7:183-4.

13. Chung E, Chen G, Alexander B, et al. Non-invasive continuous blood pressure monitoring: a review of current applications. Front Med (Lausanne) 2013;7:91-101.

14. Hager HH, Burns B. Cannulation, Artery. StatPearls

[Internet]. Treasure Island (FL): StatPearls Publishing; 2018 Jan-. Updated 2018 Feb 22.

15. Brzezinski M, Luisetti T, London MJ. Radial artery cannulation: a comprehensive review of recent anatomic and physiologic investigations. Anesth Analg 2009;109:1763-81.

16. Tobias JD, McKee C, Herz D, et al. Accuracy of the CNAP monitor, a noninvasive continuous blood pressure device, in providing beat-to-beat blood pressure measurements during bariatric surgery in severely obese adolescents and young adults. J Anesth 2014;28:861-5.

17. Meidert AS, Saugel B. Techniques for non-invasive monitoring of arterial blood pressure. Front Med (Lausanne) 2018;4:231.

18. Kuck K, Baker PD. Perioperative Noninvasive Blood Pressure Monitoring. Anesth Analg 2018;127:408-11.

19. Lehman LW, Saeed M, Talmor D, et al. Methods of blood pressure measurement in the ICU. Crit Care Med 2013;41:34-40.

20. Irving G, Holden J, Stevens R, et al. Which cuff should I use? Indirect blood pressure measurement for the diagnosis of hypertension in patients with obesity: a diagnostic accuracy review. BMJ Open 2016;6:

21. Watson S, Aguas M, Colegrove P, et al. Level of agreement between forearm and upper arm blood pressure measurements in patients with large arm circumference. $\mathrm{J}$ 
Perianesth Nurs 2017;32:15-21.

22. Schimanski K, Jull A, Mitchell N, et al. Comparison study of upper arm and forearm non-invasive blood pressures in adult Emergency Department patients. Int J Nurs Stud 2014;51:1575-84.

23. Ameloot K, Palmers PJ, Malbrain MLNG, et al. The accuracy of noninvasive cardiac output and pressure measurements with finger cuff: a concise review. Curr Opin Crit Care 2015;21:232-9.

24. Huygh J, Peeters Y, Bernards J, et al. Hemodynamic monitoring in the critically ill: an overview of current cardiac output monitoring methods. F1000Res 2016;5. doi: 10.12688/f1000research.8991.1.

25. Mehta N, Fernandez-Bustamante A, Seres T. A review of intraoperative goal-directed therapy using arterial waveform analysis for assessment of cardiac output. ScientificWorldJournal 2014;2014:702964.

26. Moura TS, Rosa SA, Germano N, et al. The accuracy of $\mathrm{PiCCO} \AA$ in measuring cardiac output in patients under therapeutic hypothermia: Comparison with transthoracic echocardiography. Med Intensiva 2018;42:92-8.

27. Herner A, Heilmaier M, Mayr U, et al. Comparison of cardiac function index derived from femoral and jugular indicator injection for transpulmonary thermodilution with the PiCCO-device: A prospective observational study. PLoS One 2018;13:e0200740.

28. Liu X, Ji W, Wang J, et al. Application strategy of PiCCO in septic shock patients. Exp Ther Med 2016;11:1335-9.

29. Zhang Z, Xu X, Yao M, et al. Use of the PiCCO system in critically ill patients with septic shock and acute respiratory distress syndrome: a study protocol for a randomized controlled trial. Trials 2013;14:32.

30. Zhang Z, Ni H, Qian Z. Effectiveness of treatment based on PiCCO parameters in critically ill patients with septic shock and/or acute respiratory distress syndrome: a randomized controlled trial. Intensive Care Med 2015;41:444-51.

31. Tsai YF, Liu FC, Yu HP. Flo'Trac/Vigileo system monitoring in acute-care surgery: current and future trends. Expert Rev Med Devices 2013;10:717-28.

32. Sugasawa Y, Hayashida M, Yamaguchi K, et al. Usefulness of stroke volume index obtained with the FloTrac/Vigileo system for the prediction of acute kidney injury after radical esophagectomy. Ann Surg Oncol 2013;20:3992-8.

33. Button D, Weibel L, Reuthebuch O, et al. Clinical evaluation of the FloTrac/Vigileo system and two established continuous cardiac output monitoring devices in patients undergoing cardiac surgery. Br J Anaesth
2007;99:329-36.

34. Suehiro K, Tanaka K, Mikawa M, et al. Improved performance of the fourth-generation FloTrac/Vigileo system for tracking cardiac output changes. J Cardiothorac Vasc Anesth 2015;29:656-62.

35. Tejedor A, Rivas E, Ríos J, et al. Accuracy of Vigileo/ Flotrac monitoring system in morbidly obese patients. J Crit Care 2015;30:562-6.

36. Lee M, Weinberg L, Pearce B, et al. Agreement in hemodynamic monitoring during orthotopic liver transplantation: a comparison of FloTrac/Vigileo at two monitoring sites with pulmonary artery catheter thermodilution. J Clin Monit Comput 2017;31:343-51.

37. Maeda T, Hattori K, Sumiyoshi M, et al. Accuracy and trending ability of the fourth generation FloTrac/Vigileo System $^{\mathrm{TM}}$ in patients undergoing abdominal aortic aneurysm surgery. J Anesth 2018;32:387-93.

38. Hattori K, Maeda T, Masubuchi T, et al. Accuracy and trending ability of the fourth-generation FloTrac/Vigileo System in patients with low cardiac index. J Cardiothorac Vasc Anesth 2017;31:99-104.

39. Kusaka Y, Ohchi F, Minami T. Evaluation of the fourthgeneration FloTrac/Vigileo System in comparison with the intermittent bolus thermodilution method in patients undergoing cardiac surgery. J Cardiothorac Vasc Anesth 2019;33:953-60.

40. Suehiro K, Tanaka K, Matsuura T, et al. The VigileoFloTrac System: arterial waveform analysis for measuring cardiac output and predicting fluid responsiveness: a clinical review. J Cardiothorac Vasc Anesth 2014;28:1361-74.

41. Romagnoli S, Ricci Z, Romano SM, et al. Flo'Trac/ Vigileo (third generation) and MostCares/PRAM versus echocardiography for cardiac output estimation in vascular surgery. J Cardiothorac Vasc Anesth 2013;27:1114-21.

42. De Backer D, Marx G, Tan A, et al. Arterial pressure-based cardiac output monitoring: a multicenter validation of the third-generation software in septic patients. Intensive Care Med 2011;37:233-40.

43. Vasdev S, Chauhan S, Choudhury M, et al. Arterial pressure waveform derived cardiac output FloTrac/Vigileo system (third generation software): comparison of two monitoring sites with the thermodilution cardiac output. J Clin Monit Comput 2012;26:115-20.

44. Maeda T, Hamaguchi E, Kubo N, et al. The accuracy and trending ability of cardiac index measured by the fourth-generation FloTrac/Vigileo system ${ }^{\mathrm{TM}}$ and the Fick method in cardiac surgery patients. J Clin Monit Comput 2019;33:767-76. 
45. Mavroudis C, Alevizos L, Stamou KM, et al. Hemodynamic monitoring during heated intraoperative intraperitoneal chemotherapy using the FloTrac/Vigileo System. Int Surg 2015;100:1033-9.

46. Sangkum L, Liu GL, Yu L, et al. Minimally invasive or noninvasive cardiac output measurement: an update. J Anesth 2016;30:461-80.

47. Donati A, Nardella R, Gabbanelli V, et al. The ability of PiCCO versus $\mathrm{LiDCO}$ variables to detect changes in cardiac index: a prospective clinical study. Minerva Anestesiol 2008;74:367-74.

48. Asamoto M, Orii R, Otsuji M, et al. Reliability of cardiac output measurements using LiDCOrapid and Flo'Trac/ Vigileo across broad ranges of cardiac output values. J Clin Monit Comput 2017;31:709-16.

49. Lamia B, Kim HK, Severyn DA, et al. Cross-comparisons of trending accuracies of continuous cardiac output measurements: pulse contour analysis, bioreactance, and pulmonary-artery catheter. J Clin Monit Comput 2018;32:33-43.

50. Nakasuji M, Okutani A, Miyata T, et al. Disagreement between fourth generation FloTrac and LiDCOrapid measurements of cardiac output and stroke volume variation during laparoscopic colectomy. J Clin Anesth 2016;35:150-6.

51. O'Loughlin E, Ward M, Crossley A, et al. Evaluation of the utility of the Vigileo FloTrac, LiDCO, USCOM and CardioQ to detect hypovolaemia in conscious volunteers: a proof of concept study. Anaesthesia 2015;70:142-9.

52. Fischer MO, Momar, de Wilde RBP, et al. Evaluation of cardiac output by 5 arterial pulse contour techniques using trend interchangeability method. Medicine 2016;95:e3530.

53. Geisen M, Ganter MT, Hartnack S, et al. Accuracy, precision, and trending of 4 pulse wave analysis techniques in the postoperative period. Journal of Cardiothoracic and Vascular Anesthesia 2018;32:715-22.

54. Saugel B, Cecconi M, Hajjar LA. Noninvasive cardiac output monitoring in cardiothoracic surgery patients: Available methods and future directions. Journal of Cardiothoracic and Vascular Anesthesia 2019;33:1742-52.

55. Saugel B, Dueck R, Wagner JY. Measurement of blood pressure. Best Pract Res Clin Anaesthesiol 2014;28:309-22.

56. Nelson MR, Stepanek J, Cevette M, et al. Noninvasive measurement of central vascular pressures with arterial tonometry: Clinical revival of the pulse pressure waveform? Mayo Clin Proc 2010;85:460-72.

57. Dueck R, Goedje O, Clopton P. Noninvasive continuous beat-to-beat radial artery pressure via TL-200 applanation tonometry. J Clin Monit Comput 2012;26:75-83.

58. Saugel B, Meidert AS, Hapfelmeier A, et al. Non-invasive continuous arterial pressure measurement based on radial artery tonometry in the intensive care unit: a method comparison study using the T-Line TL-200pro device. Br J Anaesth 2013;111:185-90.

59. Meidert AS, Huber W, Hapfelmeier A, et al. Evaluation of the radial artery applanation tonometry technology for continuous noninvasive blood pressure monitoring compared with central aortic blood pressure measurements in patients with multiple organ dysfunction syndrome. J Crit Care 2013;28:908-12.

60. Szmuk P, Pivalizza E, Warters RD, et al. An evaluation of the T-Line Tensymeter continuous noninvasive blood pressure device during induced hypotension. Anaesthesia 2008;63:307-12.

61. Sun J, Chen H, Zheng J, et al. Continuous blood pressure monitoring via non-invasive radial artery applanation tonometry and invasive arterial catheter demonstrates good agreement in patients undergoing colon carcinoma surgery. J Clin Monit Comput 2017;31:1189-95.

62. Lin WQ, Wu HH, Su CS, et al. Comparison of continuous noninvasive blood pressure monitoring by TL300 with standard invasive blood pressure measurement in patients undergoing elective neurosurgery. J Neurosurg Anesthesiol 2017;29:1-7.

63. Zayat R, Goetzenich A, Lee JY, et al. Comparison between radial artery tonometry pulse analyzer and pulsed- Doppler echocardiography derived hemodynamic parameters in cardiac surgery patients: a pilot study. PeerJ 2017;5:e4132.

64. Zayat R, Drosos V, Schnoering H, et al. Radial artery tonometry to monitor blood pressure and hemodynamics in ambulatory left ventricular assist device patients in comparison with doppler ultrasound and transthoracic echocardiography: A pilot study. Artificial Organs 2019;43:242-53.

65. Saugel B, Fassio F, Hapfelmeier A, et al. The T-Line TL200 system for continuous non-invasive blood pressure measurement in medical intensive care unit patients. Intensive Care Med 2012;38:1471-7.

66. Cheng HM, Lang D, Tufanaru C, et al. Measurement accuracy of non-invasively obtained central blood pressure by applanation tonometry: A systematic review and metaanalysis. Int J Cardiol 2013;167:1867-76.

67. Teboul JL, Saugel B, Cecconi M, et al. Less invasive hemodynamic monitoring in critically ill patients. Intensive Care Med 2016;42:1350-9.

68. Kakuta N, Tsutsumi YM, Murakami C, et al. Effectiveness 
of using non-invasive continuous arterial pressure monitoring with ClearSight in hemodynamic monitoring during living renal transplantation in a recipient : a case report. J Med Invest 2018;65:139-41.

69. Gellert G, Bramlage P. Use of the ClearSight ${ }^{\circledR}$ System for continuous noninvasive hemodynamic monitoring during heart valve interventions: Review of the literature and single-site experience. Heart Surg Forum 2018;21:E476-E83.

70. Rogge DE, Nicklas JY, Schön G, et al. Continuous noninvasive arterial pressure monitoring in obese patients during bariatric surgery: An evaluation of the vascular unloading technique (Clearsight system). Anesth Analg 2019;128:477-83.

71. Meidert AS, Nold JS, Hornung R, et al. The impact of continuous non-invasive arterial blood pressure monitoring on blood pressure stability during general anaesthesia in orthopaedic patients. Eur J Anaesthesiol 2017;34:716-22.

72. Sakai Y, Tsutsumi YM, Oyama T, et al. Noninvasive continuous blood pressure monitoring by the ClearSight system during robot-assisted laparoscopic radical prostatectomy. J Med Invest 2018;65:69-73.

73. Martina JR, Westerhof BE, van Goudoever J, et al. Noninvasive continuous arterial blood pressure monitoring with Nexfin. Anesthesiology 2012;116:1092-103.

74. Schattenkerk DWE, van Lieshout JJ, van den Meiracker AH, et al. Nexfin noninvasive continuous blood pressure validated against Riva-Rocci/Korotkoff. Am J Hypertens 2009;22:378-83.

75. Ameloot K, de Vijver KV, Broch O, et al. Nexfin noninvasive continuous hemodynamic monitoring: Validation against continuous pulse contour and intermittent transpulmonary thermodilution derived cardiac output in critically ill patients. ScientificWorldJournal 2013;2013:1-11.

76. van der Spoel AGE, Voogel AJ, Folkers A, et al. Comparison of noninvasive continuous arterial waveform analysis (Nexfin) with transthoracic Doppler echocardiography for monitoring of cardiac output. J Clin Anesth 2012;24:304-9.

77. Bubenek-Turconi SI, Craciun M, Miclea I, et al. Noninvasive continuous cardiac output by the Nexfin before and after preload-modifying maneuvers: A comparison with intermittent thermodilution cardiac output. Anesth Analg 2013;117:366-72.

78. Aritürk C, Acil M, H. U, et al. Is the Nexfin finger cuff method for cardiac output measurement reliable during coronary artery bypass grafting? A prospective comparison with the echocardiography and FloTrac/Vigileo methods. Turk J Med Sci 2016;46:291-5.

79. Peyton PJ, Chong SW. Minimally invasive measurement of cardiac output during surgery and critical care: A meta-analysis of accuracy and precision. Anesthesiology 2010;113:1220-35.

80. Stover JF, Stocker R, R. L, et al. Noninvasive cardiac output and blood pressure monitoring cannot replace an invasive monitoring system in critically ill patients. BMC Anesthesiology 2009;9:6.

81. Fortin J, Wellisch A, Maier K. CNAP - Evolution of Continuous Non-Invasive Arterial Blood Pressure Monitoring. Biomed Tech (Berl) 2013;58:1-2.

82. Fortin J, Marte W, Grüllenberger R, et al. Continuous non-invasive blood pressure monitoring using concentrically interlocking control loops. Comput Biol Med 2006;36:941-57.

83. Biais M, Vidil L, Roullet S, et al. Continuous non-invasive arterial pressure measurement: Evaluation of CNAP device during vascular surgery. Ann Fr Anesth Reanim 2010;29:530-5.

84. Schramm C, Huber A, Plaschke K. The accuracy and responsiveness of continuous noninvasive arterial pressure during rapid ventricular pacing for transcatheter aortic valve replacement. Anesth Analg 2013;117:76-82.

85. Greiwe G, Tariparast PA, Behem C, et al. Is applanation tonometry a reliable method for monitoring blood pressure in morbidly obese patients undergoing bariatric surgery? Br J Anaesth 2016;116:790-6.

86. Smolle KH, Schmid M, Prettenthaler H, et al. The accuracy of the $\mathrm{CNAP} \circledast$ device compared with invasive radial artery measurements for providing continuous noninvasive arterial blood pressure readings at a medical intensive care unit: A method-comparison study. Anesth Analg 2015;121:1508-16.

87. Wagner JY, Prantner JS, Meidert AS, et al. Noninvasive continuous versus intermittent arterial pressure monitoring: evaluation of the vascular unloading technique (CNAP device) in the emergency department. Scand J Trauma Resusc Emerg Med 2014;22:8.

88. Biais M, Stecken L, Martin A, et al. Automated, continuous and non-invasive assessment of pulse pressure variations using CNAP system. J Clin Monit Comput 2017;31:685-92.

89. Dewhirst E, Corridore M, Klamar J, et al. Accuracy of the CNAP monitor, a noninvasive continuous blood pressure device, in providing beat-to-beat blood pressure readings in the prone position. J Clin Anesth 2013;25:309-13.

90. Jagadeesh AM, Singh NG, Mahankali S. A comparison 
of a continuous noninvasive arterial pressure (CNAP $\left.{ }^{\mathrm{TM}}\right)$ monitor with an invasive arterial blood pressure monitor in the cardiac surgical ICU. Ann Card Anaesth 2012;15:180-4.

91. Kim SH, Lilot M, Sidhu KS, et al. Accuracy and precision of continuous noninvasive arterial pressure monitoring compared with invasive arterial pressure: A systematic review and meta-analysis. Anesthesiology 2014;120:1080-97.

92. Vos JJ, Poterman M, Mooyaart EAQ, et al. Comparison

Cite this article as: Pour-Ghaz I, Manolukas T, Foray N, Raja J, Rawal A, Ibebuogu UN, Khouzam RN. Accuracy of noninvasive and minimally invasive hemodynamic monitoring: where do we stand? Ann Transl Med 2019;7(17):421. doi: 10.21037/atm.2019.07.06 of continuous non-invasive finger arterial pressure monitoring with conventional intermittent automated arm arterial pressure measurement in patients under general anaesthesia. Br J Anaesth 2014;113:67-74.

93. Wagner JY, Grond J, Fortin J, et al. Continuous noninvasive cardiac output determination using the CNAP system: evaluation of a cardiac output algorithm for the analysis of volume clamp method-derived pulse contour. J Clin Monit Comput 2016;30:487-93. 